\title{
DUAL ORTHOGONAL SERIES: AN ABSTRACT APPROACH
}

\author{
BY ROBERT P. FEINERMAN ${ }^{1}$ AND ROBERT B. KELMAN ${ }^{2}$ \\ Communicated by Alberto Calderón, March 11, 1975
}

Introduction. Problems in dual orthogonal series can usually be put in the following form: Given $\left\{\phi_{n}\right\}$, a complete orthonormal sequence in $L^{2}[0,1]$, two sequences $\left\{a_{n}\right\}$ and $\left\{b_{n}\right\}$ of nonnegative constants, a point $c$ in $(0,1)$ and an $f$ in $L^{2}[0,1]$, find a sequence $\left\{j_{n}\right\} \in l^{2}$ such that

$$
\sum_{n=1}^{\infty} j_{n} \psi_{n}=f \quad \text { (in the } L^{2}[0,1] \text { norm) }
$$

where the base functions $\psi_{n}$ are defined by

$$
\psi_{n}= \begin{cases}a_{n} \phi_{n} & \text { in }(0, c), \\ b_{n} \phi_{n} & \text { in }(c, 1) .\end{cases}
$$

Some related problems concern the uniqueness and approximation of $\left\{j_{n}\right\}$ (when solutions to equation (1) exist) and the completeness of $\left\{\psi_{n}\right\}$ (even when solutions to equation (1) do not necessarily exist).

In our abstract approach we proceed as follows: Let $R$ be a real, separable Hilbert space and let $P$ and $Q$, subspaces of $R$, be orthogonal complements. $p$ and $q$ are the projection operators onto $P$ and $Q$ respectively. Let $\left\{\psi_{n}\right\}$ be a complete, orthonormal sequence in $R$, and let $\left\{a_{n}\right\}$ and $\left\{b_{n}\right\}$ be sequences of nonnegative constants. The general problem is: Given $f \in R$ find a sequence $\left\{j_{n}\right\} \in l^{2}$ such that

$$
\sum_{n=1}^{\infty} j_{n} \psi_{n}=f \quad(\text { in the norm of } R \text { ) }
$$

where the base functions $\psi_{n}$ are now defined by

$$
\psi_{n}=a_{n} p\left(\phi_{n}\right)+b_{n} q\left(\phi_{n}\right) \text {. }
$$

AMS (MOS) subject classifications (1970). Primary 44A52, 44A64.

Key words and phrases. Dual series, basis, completeness.

${ }^{1}$ Partially supported by Grant No. 10167 of the CUNY Faculty Research Award Program.

${ }^{2}$ Partially supported by U.S. Army Research Office under Grant No. DAH C04 74 G0140. 
In this note, we announce some results about the existence, uniqueness and approximation of $\left\{j_{n}\right\}$ (when solutions to equation (2) exist) and about the completeness of $\left\{\psi_{n}\right\}$ (even when solutions to (2) do not necessarily exist). The proofs, being lengthy and involved, will be published elsewhere (see [1] and [2]).

\section{Existence and uniqueness.}

THEOREM 1. If $\left\{a_{n}\right\}$ and $\left\{b_{n}\right\}$ are sequences of positive constants, one of which is bounded above zero, and if, as $n$ approaches infinity, $b_{n} / a_{n}$ converges to a positive limit, then, for any $f$ in $R$, equation (2) has a unique solution $\left\{j_{n}\right\} \in l^{2}$. In fact, $\left\{j_{n} a_{n}\right\}$ and $\left\{j_{n} b_{n}\right\}$ are in $l^{2}$.

This result is applicable to all dual Sturm-Liouville series associated with mixed boundary conditions of the second and third kind (see the appendix of [2] for a definition of the various boundary conditions and [3] for a general background of the relation of the boundary conditions to dual orthogonal series).

To illustrate the application of this theorem we introduce the following potential problem: Let $h_{1}$ and $h_{2}$ be nonnegative constants. For a given $f(x)$ in $L^{2}[0, \pi]$, we seek the potential $u(x, y)$ in $\{0<x<\pi ; y>0\}$ with

(3a) $u(x, y)$ bounded at infinity,

(3b) $u_{x}(0, y)=0$ for $y>0$,

(3c) $u(\pi, y)=0$ for $y>0$,

(3d) $u_{y}(x, 0)=h_{1} u(x, 0)-f(x)$ for $0<x<c$,

(3e) $u_{y}(x, 0)=h_{2} u(x, 0)-f(x)$ for $c<x<\pi$.

Conditions (3a), (3b) and (3c), when used with the method of separation of variables, imply $u$ can be expressed by $\Sigma j_{n}(\cos (n-1 / 2) x)(\exp (-n+1 / 2) y)$.

For conditions (3d) and (3e) to be satisfied we must have

(4a) $\Sigma j_{n}\left(n-1 / 2+h_{1}\right) \sqrt{\pi / 2} \cos (n-1 / 2) x=\sqrt{\pi / 2} f(x)$ for $0<x<c$,

(4b) $\Sigma j_{n}\left(n-1 / 2+h_{2}\right) \sqrt{\pi / 2} \cos (n-1 / 2) x=\sqrt{\pi / 2} f(x)$ for $c<x<\pi$.

If we set $a_{n}=n-1 / 2+h_{1}, b_{n}=n-1 / 2+h_{2}$ and $\phi_{n}=\sqrt{\pi / 2} \cos (n-1 / 2) x$, then the hypothesis of Theorem 1 is satisfied, so that there exists a unique sequence $\left\{j_{n}\right\} \in l^{2}$ satisfying the dual trigonometric equation (4a) and (4b).

In many cases, we have $a_{1}$ or $b_{1}$ equal to zero, corresponding to an eigenvalue associated with $\phi_{1}$ being zero. This occurs when the boundary conditions associated with $\left\{\phi_{n}\right\}$ (e.g., (3b) and (3c) in the above example) are Neumann, periodic or singular. These cases are covered by Theorem 2 in 
which $p_{k j}$ and $q_{k j}$ denote respectively the inner products $\left(p \phi_{k}, \phi_{j}\right)$ and $\left(q \phi_{k}, \phi_{j}\right)$.

THEOREM 2. Let $\left\{a_{n}\right\}$ be a positive sequence, bounded above zero. Let $\left\{b_{n}\right\}$ be a nonnegative sequence such that, for some $N, b_{n}>0$ for $n>N$ and such that $a_{n} / b_{n}$ converges to a positive limit as $n$ approaches infinity. Let the subspace spanned by the $N$-dimensional vectors $\left(p_{k_{1}}, p_{k_{2}}, p_{k_{3}}, \ldots, p_{k N}\right)$, $k=1,2,3, \ldots$, have dimension $N$. Then equation (2) has a unique solution $\left\{j_{n}\right\} \in l^{2}$. In fact, $\left\{j_{n} a_{n}\right\}$ and $\left\{j_{n} b_{n}\right\}$ are also in $l^{2}$. (The roles of $\left\{a_{n}\right\}$ and $\left\{b_{n}\right\}$ can be switched with a corresponding switch of $q_{k n}$ for $p_{k n}$.)

This theorem is applicable in the potential problem that arises in seeking the steady temperature $u(r, \theta)$ in a thermally homogeneous sphere with prescribed heat flux through the top half of the spherical surface and Newtonian heat loss through the bottom half.

Completeness and approximation. We now establish the completeness of $\left\{\psi_{n}\right\}$ under hypotheses weaker than those of Theorems 1 or 2 (here $a_{n} / b_{n}$ need not converge to a positive limit).

Theorem 3. Let $\left\{a_{n}\right\}$ be a positive sequence. Let $\left\{b_{n}\right\}$ be a nonnegative sequence such that either

(i) $b_{n}>0$ for all $n$, or

(ii) there is an $N$ such that $b_{n}=0$ for $n=1,2, \ldots, N$, while $b_{n}>0$ for $n>N$ and the subspace spanned by the $N$-dimensional vectors $\left(p_{k_{1}}, p_{k_{2}}\right.$, $\left.\ldots, p_{k N}\right) k=1,2,3, \ldots$ has dimension $N$. Then $\left\{\psi_{n}\right\}$ is complete and (finitely) linearly independent in $R$. (The roles of $\left\{a_{n}\right\}$ and $\left\{b_{n}\right\}$ can be switched with a corresponding switch of $q_{k n}$ for $p_{k n}$.)

It can be easily shown that for any given $f \in R$ and for any $N$, the solution to the system of equations

$$
\sum_{n=1}^{N}\left(\psi_{k}, \psi_{n}\right) j_{n}=\left(f, \psi_{k}\right), \quad k=1,2, \ldots, N
$$

minimizes the expression $\left\|\Sigma_{n=1}^{N} j_{n} \psi_{n}-f\right\|$. Combining this observation with the previous theorem we have the first part of

THEOREM 4. Let the hypothesis of Theorem 3 hold and let $f \in R$ be given. For each $N$, let $j_{1}(N), j_{2}(N), \ldots, j_{N}(N)$ be the (unique) solution to the system of equations (5). Then $\left\|\left(\Sigma_{n=1}^{N} j_{n}(N) \psi_{n}\right)-f\right\|$ goes to zero as $N$ approaches infinity. If, in addition, the hypotheses of Theorem 1 or 2 hold, 
$\Sigma_{n=1}^{N}\left(j_{n}(N)-j_{n}\right)^{2}$ goes to zero as $N$ approaches infinity, where $\left\{j_{n}\right\}$ is the solution to equation (2).

These last two theorems are applied in considering the potential problem involving the temperature in a sphere having prescribed temperature in the top half and Newtonian heat loss through the lower half. (In fact, a survey of some seventy papers involving dual orthogonal series shows that these last two theorems are sufficiently general to apply to all of them.)

\title{
REFERENCES
}

1. R. P. Feinerman and R. B. Kelman, The convergence of least square approximations for dual orthogonal series, Glasgow Math. J. 15 (1974), 82-85.

2. R. B. Kelman and R. P. Feinerman, Dual orthogonal series, SIAM J. Math. Anal. 5 (1974), 489-502.

3. I. N. Sneddon, Mixed boundary value problems in potential theory, NorthHolland, Amsterdam; Interscience, New York, 1966. MR 35 \#6853.

DEPARTMENT OF MATHEMATICS, LEHMAN COLLEGE, CITY UNIVERSITY OF NEW YORK, BRONX, NEW YORK 10468

DEPARTMENT OF COMPUTER SCIENCE, COLORADO STATE UNIVERSITY, FORT COLLINS, COLORADO 80523

BULLETIN OF THE

AMERICAN MATHEMATICAL SOCIETY

Volume 81, Number 4, July 1975

\section{CONFORMAL GEOMETRY IN HIGHER DIMENSIONS. I}

\author{
BY R. S. KULKARNI
}

Communicated ${ }^{1}$ February 24, 1975

Conformally Euclidean manifolds are one type of higher dimensional generalization of Riemann surfaces. They are studied and classified here from that point of view (cf. [2] and [3]).

1. Definition 1.1. A conformal structure on a manifold $M$ is a covering $\left\{U_{\alpha}\right\}$ together with a metric $g_{\alpha}$ on $U_{\alpha}$ such that whenever $U_{\alpha} \cap U_{\beta} \neq \varnothing, g_{\alpha}$ and $g_{\beta}$ are conformally related on $U_{\alpha} \cap U_{\beta}$.

AMS (MOS) subject classifications (1970). Primary 53A30.

1 Originally received July 24, 1974. 